\title{
EVALUACIÓN DE CAPAS DE BLOQUEO EN CELDAS SOLARES SENSIBILIZADAS DE ÓXIDO DE TITANIO NANOESTRUCTURADO
}

\author{
Russell Nazario $^{\mathrm{a}}$, Williams Savero Torres ${ }^{\mathrm{a}, \mathrm{b}}$, Eduardo Palacios ${ }^{\mathrm{a}}$ y María Quintana ${ }^{\mathrm{a}}$ \\ mquintana@uni.edu.pe
}

\begin{abstract}
RESUMEN
En las celdas Grätzel convencionales, sobre un sustrato conductor (FTO), se deposita el electrodo, una capa mesoporosa de $\mathrm{TiO}_{2} 20 \mu \mathrm{m}$, preparados por el método doctor blade, con un tratamiento térmico a $450{ }^{\circ} \mathrm{C}$, sensibilizado con el colorante (D7). En el presente artículo se agregó una capa adicional de $\mathrm{TiO}_{2}$ entre el FTO y el electrodo, ya que el electrodo tiene una gran porosidad y no tiene un buen contacto con el FTO, por eso se propuso agregar una capa más compacta que ayude a la transferencia del electrón del electrodo al FTO y evita el contacto directo entre el FTO y electrolito. De ese modo se evita la recombinación del electrón del FTO con el hueco del electrolito. Posteriormente, se realizó caracterizaciones para determinar el espesor óptimo de la capa de bloqueo y analizar la morfología del electrodo mediante Microscopía de Barrido Electrónico. Finalmente, se determinó la curva I-V para analizar su eficiencia, con una radiación de $1000 \mathrm{~W} / \mathrm{m}^{2}$ se alcanzó una eficiencia de 2,39\%.
\end{abstract}

Palabras clave: Electrodo, sensibilizado, capa de bloqueo, electrolito, recombinación.

\section{EVALUATION OF BLOCKING LAYERS SENSITIZED SOLAR CELLS OF NANOSTRUCTURED TITANIUM OXIDE}

\begin{abstract}
In conventional Grätzel cells, the conductive substrate (FTO) on the electrode, a mesoporous $\mathrm{TiO}_{2}$ layer $\sim$ um, prepared by the doctor blade method, with a heat treatment at $450{ }^{\circ} \mathrm{C}$, the dye-sensitized deposited (D7). In this article an additional $\mathrm{TiO}_{2}$ layer between the FTO and the electrode is added, because the electrode has a high porosity and don't has a good contact with the FTO, so we suggested adding a compact layer that helps transfer electron of electrode to FTO and prevents direct contact between the FTO and electrolyte. Thus electron recombination is prevented by the FTO hollow electrolyte. Subsequently certain characterizations are performed to determine the thickness of the block and to analyze the morphology of the electrode by Scanning Electron Microscopy. Finally the I-V curve was we determined to analyze its efficiency with a radiation of $1000 \mathrm{~W} / \mathrm{m}^{2}$ efficiency of $2,39 \%$ was reached.
\end{abstract}

Key words: Electrode, sensitized, blocking layer, electrolyte, recombination.

\section{INTRODUCCIÓN}

La contaminación en estos tiempos ha alterado el balance natural de los niveles de $\mathrm{CO}_{2}$ en la atmósfera. La absorción infrarroja de la radiación solar y la longevidad del $\mathrm{CO}_{2}$ atmosférico están causando el efecto invernadero. Entonces los retos más grandes que encara

a Laboratorio de Películas Delgadas, Facultad de Ciencias, Universidad Nacional de Ingeniería, Lima, Perú, Avenida Túpac Amaru ํ2 210 Rímac.

b Laboratoire de Nanostructures et Magnetisme/SP2M/ INAC/CEA. Grenoble- France 
los tiempos actuales son el incremento global de la demanda de energía y el control del nivel de emisiones de $\mathrm{CO}_{2}$. Por lo tanto, uno de los objetivos de la humanidad es el desarrollo de fuentes de energías alternativas como las energías renovables, buscando la protección del medio ambiente ${ }^{1,2}$. La energía renovable es fuente de la naturaleza, siendo virtualmente inagotables, no sólo porque contienen una inmensa cantidad de energía sino porque además, son capaces de regenerarse por medios naturales ${ }^{3}$. Un tipo de energía renovable es la energía solar y una forma de aprovechar este tipo de energía es la generación de fuentes fotovoltaica como las celdas solares.

La tecnología fotovoltaica ha ido evolucionando en diferentes etapas. La primera etapa comenzó en 1954 con celdas solares a base de silicio con una eficiencia del 6\%4. Las celdas solares de silicio se encuentran entre las más difundidas en la actualidad, constituyendo cerca del $82 \%$ del mercado fotovoltaico ${ }^{5}$. Sin embargo, su proceso de fabricación es aún elevado. La segunda generación se basa en múltiples capas de semiconductores de uniones $\mathrm{p}-\mathrm{n}^{9}$. Por ejemplo las basadas en la unión $C d T e / C d S$ han alcanzado eficiencias superiores a un $16 \%$. Los materiales usados en estas celdas son muy costosos y no se encuentran ampliamente difundidos a escala comercial para aplicaciones terrestres ${ }^{4}$, habiéndose reportado, a base de estos materiales, con eficiencias de hasta $10,4 \%{ }^{4}$. La tercera generación son las Celdas Solares Sensibilizados con Colorante (DSC) o también llamadas celdas Grätzel. En 1991, en los Laboratorios de la Universidad de Lausanne en Suiza, Michael Grätzel y Brian O'Regan provocaron una revolución en la utilización de las celdas fotoelectroquímicas, al utilizar un electrodo poroso de $\mathrm{TiO}_{2}$ nanocristalino que recubrieron con una monocapa de un compuesto orgánico con rutenio que actúa como sensibilizador ${ }^{8}$. Este tipo de celdas en comparación con las generaciones anteriores son más económicas y tiene componentes no contaminante, pero su desventaja es una menor eficiencia en comparación con la antiguas generaciones ${ }^{9}$, por ello se investiga en optimizar su eficiencia probando diferente elementos o técnicas que ayuden con este propósito. El fin de este trabajo de investigación es la de ayudar a aumentar la eficiencia con la capa de bloque que optimice el desempeño de la celda, los otro elementos de la celda, se trabajaron con valores establecidos.

El objetivo de un dispositivo fotovoltaico (celda solar) es el de convertir la energía solar en energía eléctrica, implicando para ello diversos procesos fundamentales que explicaremos a continuación ${ }^{10,11}$. La celda Grätzel usa semiconductores como el $\mathrm{TiO}_{2}$, pero como este material sólo absorbe la radicación solar en el rango ultravioleta, se coloca un colorante (sensibilizador) que amplía su respuesta espectral, haciendo que sus moléculas absorban fotones de la región del espectro visible. Este último proceso es llamado la sensibilización del semiconductor ${ }^{12}$. La operación de una celda solar general se divide en varias etapas ${ }^{11}$ : La primera es la absorción de la luz por el colorante $\mathrm{S}$ que conduce a la formación del estado electrónicamente excitado del colorante:

$$
\mathrm{S}+\mathrm{hv} \rightarrow \mathrm{S}^{*} \quad \text { Absorción }
$$

El colorante al ser excitado produce excitones (par electrón libre-hueco), solamente los electrones se inyectan en la banda de conducción del semiconductor $\mathrm{TiO}_{2}$ y los huecos al electrolito ${ }^{13}$.

$$
\mathrm{S}^{*} \rightarrow \mathrm{S}^{+}+\mathrm{e}_{\left(T_{i} O_{2}\right)}^{-} \text {Inyección de carga }
$$


Mientras que estos huecos son llevados por el electrolito (par rédox yoduro/ triyoduro, $\mathrm{I}^{-} / \mathrm{I}_{3}^{-}$) hacia el contraelectrodo (cátodo), en este caso el Pt, donde el electrolito dona un electrón al colorante $\mathrm{S}$ ayudando a que se regenere.

$$
2 \mathrm{~S}^{+}+3 \mathrm{I}^{-} \rightarrow 2 \mathrm{~S}^{+} \mathrm{I}_{3}^{-} \quad \text { Regeneración de } \mathrm{S}
$$

Al unir la celda a un circuito exterior los electrones llegan al contraelectrodo, teniendo un proceso cíclico en la celda

Cátodo:

$$
\mathrm{I}_{3}^{-}+2 \mathrm{e}_{(P t)}^{-} \rightarrow 3 \mathrm{I}^{-} \quad \text { Regeneración de } \mathrm{I}^{-}
$$

Si sólo tomaran lugar las reacciones citadas, el efecto global de la radiación solar celda:

Donde:

$$
\mathrm{e}_{(P t)}^{-}+\mathrm{hv} \rightarrow \mathrm{e}_{\left(T i O_{2}\right)}^{-}
$$

hv: Fotón incidente en la celda solar

$\mathrm{e}_{\left(T \mathrm{TO}_{2}\right)}^{-}$: Electrón que se desplaza por el $\mathrm{TiO}_{2}$

$\mathrm{e}_{\left(P_{t}\right)}^{-}$: Electrón que se desplaza por el platino

En este trabajo se utilizó un colorante orgánico D7 cuya estructura molecular es $\mathrm{C}_{46} \mathrm{H}_{33} \mathrm{O}_{2} \mathrm{~N}_{2} \mathrm{~S}_{1}{ }_{1}$ , cuyo rango de absorción espectral (figura 1), llega hasta los $540 \mathrm{~nm}$. Es decir, absorbe el rango ultravioleta y parte del visible. Este es un colorante orgánico, pero en las celdas solares los de mejor rendimiento son los metal-orgánico, que contienen el rutenio en su estructura, pero son muy caros, en comparación con los colorantes orgánicos.
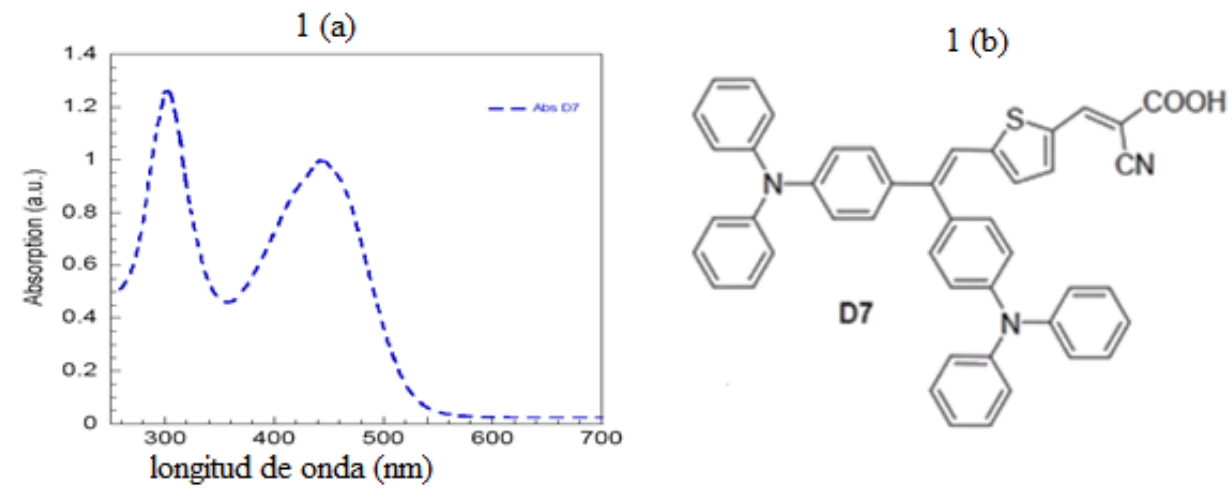

Figura 1. . (a) Gráfico de absorción (u.a.) versus longitud de onda $n m$ del colorante $D 7$.

(b) Estructura molecular del colorante D ${ }^{14}$. 
Hay tres casos frecuentes de vías de recombinación dentro de la celda. Uno de ellos implica la recombinación del electrón $e_{\mathrm{TiO}_{2}}^{-}$con el colorante oxidado antes de que el colorante pueda ser regenerado por el par rédox: ${ }^{15}$

$$
e_{\mathrm{TiO}_{2}}^{-}+D^{+} \rightarrow D
$$

Los otros dos casos de recombinación son la recombinación del $e_{\mathrm{TiO}_{2}}^{-}$con las especies rédox oxidados

$$
e_{\mathrm{TiO}_{2}}^{-}+R^{+} \rightarrow R
$$

y la recombinación del electrón en el sustrato de FTO $e_{F T O}^{-}$con las especies rédox oxidados:

$$
e_{F T O}^{-}+R^{+} \rightarrow R
$$

La última recombinación puede ser inhibida por la aplicación de una capa de bloqueo depositada sobre el FTO para cubrir los espacios en el que el sustrato FTO entra en contacto directo con la solución que contiene al par rédox ${ }^{15,16}$.

\section{PARTE EXPERIMENTAL}

Para conseguir la solución de $\mathrm{TiO}_{2}$ se usó el método Sol-Gel ${ }^{17}$. En $80 \mathrm{~mL}$ de agua destilada se mezcla 0,61 mL de ácido nítrico (HNO_3) al 70\%, luego la solución se agita empleando un agitador magnético alrededor de 15 minutos. Posteriormente, se colocó $4 \mathrm{~mL}$ de isopropóxido de titanio a una temperatura de $50{ }^{\circ} \mathrm{C}$ durante 15 minutos, luego se elevó la temperatura hasta $100^{\circ} \mathrm{C}$, y se siguió agitando 2 horas 45 minutos.

Usando la técnica del rociado Pirolítico ${ }^{18}$, como se observa en la figura 2, se obtiene las capas de bloqueo. Para este paso se utilizó el equipo del Rociado Pirolítico. Para esto se alistó la solución de $\mathrm{TiO}_{2}$ preparada con anticipación. Para conseguir estas películas o capas de bloqueo los parámetros establecidos son: temperatura de $250^{\circ} \mathrm{C}$ y una presión de $25 \mathrm{Bar}^{18}$, (el espesor de la capa de bloqueo se controla con el número de pasadas).

El electrodo se obtuvo a partir de una mezcla de $20 \%$ de nanopartículas de $\mathrm{TiO}_{2}$ con $80 \%$ de etanol puro, homogenizadas por agitación por un tiempo de 12 horas. Con esta mezcla se hizo un recubrimiento sobre la capa de bloqueo cubriendo un área de $0,5 x 0,5 \mathrm{~cm}$ delimitada por cinta adhesiva (cinta mágica) pegada sobre la superficie conductora del sustrato; se usó una bagueta para extender esta pasta, este procedimiento es el llamado método del doctor Blade ${ }^{19}$, obteniendo así un recubrimiento del material semiconductor. 


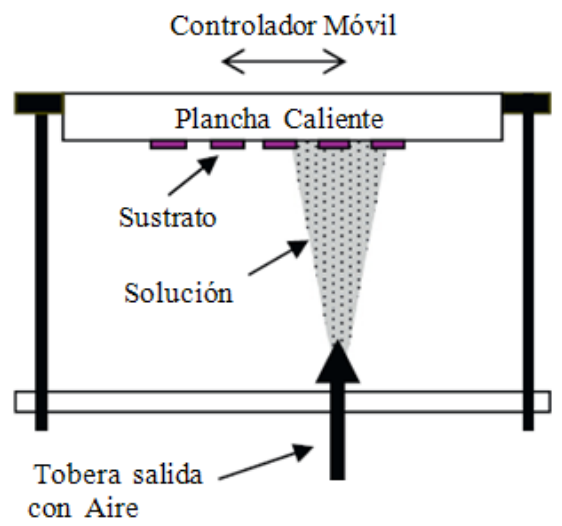

Figura 2. Esquema del instante que se realiza el rociado pirolítico con una solución de $\mathrm{TiO}_{2}$ sobre el sustrato conductor ${ }^{18}$

Seguidamente, este electrodo se sometió a un tratamiento térmico a $450^{\circ} \mathrm{C}$ por $30 \mathrm{~min}$ para remover los aditivos orgánicos y también para conseguir la sinterización entre las nanopartículas del semiconductor y del sustrato. Para el electrolito empleamos 0,5 M LiI, 0,05 $\mathrm{M} \mathrm{I}_{2}, 0.5 \mathrm{M}$ 4-tert-butylpyridine (4-TBP), en $5 \mathrm{~mL}$ de 3-methoxypropionitrile (MPN) como electrolito de yoduro / triyoduro $\left(\mathrm{I}^{-} / \mathrm{I}_{3}^{-}\right) 20$. El contra electrodo está compuesto por una placa de sustrato conductor, el cual es recubierto con un material catalítico $\left(\mathrm{H}_{2} \mathrm{C}_{16} \mathrm{Pt} .6 \mathrm{H}_{2} \mathrm{O}\right)$. Una vez aplicada la solución de platino, el contraelectrodo es llevado al horno a una temperatura de $450^{\circ} \mathrm{C}$ por un tiempo de 15 minutos y se deja enfriar lentamente dentro del horno. Después de la preparación de las películas nanoporosas de $\mathrm{TiO}_{2}$ cuando esté a una temperatura de $80^{\circ} \mathrm{C}$, estas fueron sumergidas en una solución de etanol puro con $0,3 \mathrm{mM}$ del colorante orgánico D7, por un tiempo de 14 horas en oscuridad ${ }^{14}$; hasta este punto del proceso se observa en la figura 3 (para eso se cubrió el frasco con papel aluminio). Luego de la sensibilización los electrodos fueron rociados con etanol para remover el exceso de colorante. Luego los electrodos coloreados son directamente usados para ensamblar la celda.

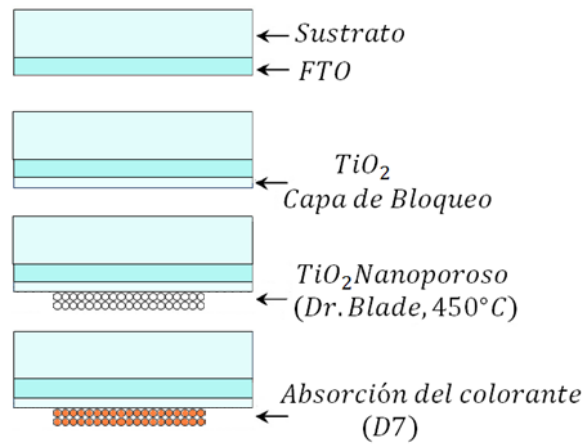

Figura 3. Esquema del procedimiento hasta conseguir el electrodo sensibilizado con el colorante $\mathrm{D} 7^{18}$. 


\section{Medidas de la celda solar}

La DSC se conecta a un circuito exterior como se muestra en la figura 4. Si la resistencia del circuito exterior es cero, la celda se pone en cortocircuito (SC) y la corriente alcanza un valor máximo I_SC, mientras que el voltaje es cero. A la inversa, si la resistencia del circuito exterior es infinita, la celda está en circuito abierto $(\mathrm{OC})$ y el voltaje máximo $\mathrm{V}_{\mathrm{OC}}$ se da cuando la corriente es cero $^{21}$.

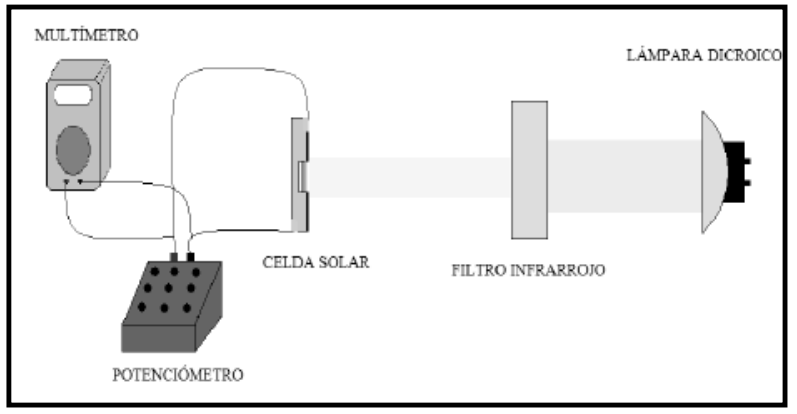

Figura 4. Esquema del proceso de obtención de la curva voltaje vs corriente de la celda solar.

Siendo la potencia igual el producto de la corriente I con el voltaje, la curva I-V, indica que hay un potencial máximo para un punto único de $\mathrm{I}-\mathrm{V}$, como indica la figura 5 ; entonces la potencia de salida máxima $\mathrm{P}_{\max }$ de un DSC es igual al producto de la corriente de punto de máxima potencia $\mathrm{I}_{\mathrm{MPP}} \mathrm{y}$ del voltaje de punto de máxima potencia $\mathrm{V}_{\mathrm{MPP}}$, como se expresa en la siguiente ecuación ${ }^{21,22}$.

$$
P_{M A X}=V_{M P P} I_{M P P}
$$

El rendimiento de la celda Grätzel es crucial y siempre han sido diseñados utilizando los parámetros: voltaje de circuito abierto $\mathrm{V}_{\mathrm{OC}}$, factor de forma FF y la corriente de corto circuito $\left(\mathrm{I}_{\mathrm{SC}}\right)$, y esto se puede apreciar en una curva I-V como se muestra en la figura 5.

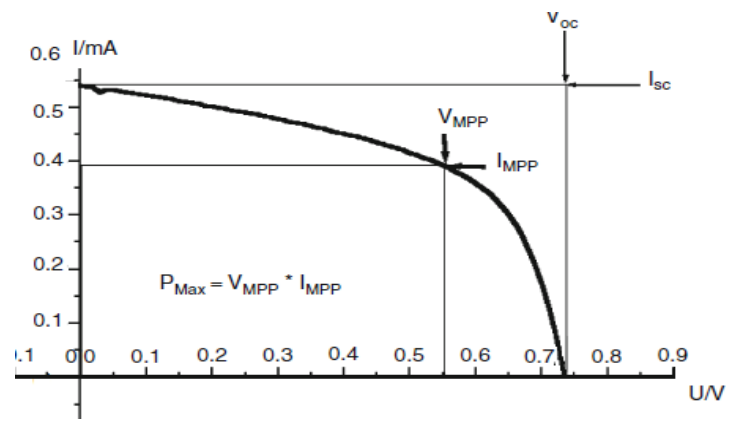

Figura 5. Curva característica de una celda solar. Curva I versus V 
La eficiencia $(\eta)$ se expresa como usando la ecuación ${ }^{22}$ :

$$
\eta=\frac{P_{M A X}}{P_{\text {in }}}
$$

El Factor Forma (FF) se expresa

$$
F F=\frac{P_{M A X}}{V_{O C} I_{S C}}
$$

Dónde:

$V_{O C}$ : Voltaje máximo obtenido a corriente cero.

$I_{S C} \quad$ : La corriente de cortocircuito.

$P_{\text {in }} \quad$ : Intensidad de radiación solar.

$I_{M P P} \quad$ : Corriente máxima.

$V_{M P P} \quad$ : Voltaje máximo.

Una vez construida la celda se realizan las conexiones adecuadas para observar el voltaje en circuito abierto $\left(\mathrm{V}_{\mathrm{OC}}\right)$, la corriente en corto circuito $\left(\mathrm{I}_{\mathrm{SC}}\right)$ y la potencia máxima $\left(\mathrm{P}_{\mathrm{m}}\right)$ como se muestra en la figura 4 haciendo variar la resistencia para determinar los datos de corriente y voltaje usando la ley Ohm.

\section{RESULTADOS Y DISCUSIÓN}

Caracterización de la superficie del electrodo $\mathrm{TiO}_{2}$ con microscopio de barrido electrónico

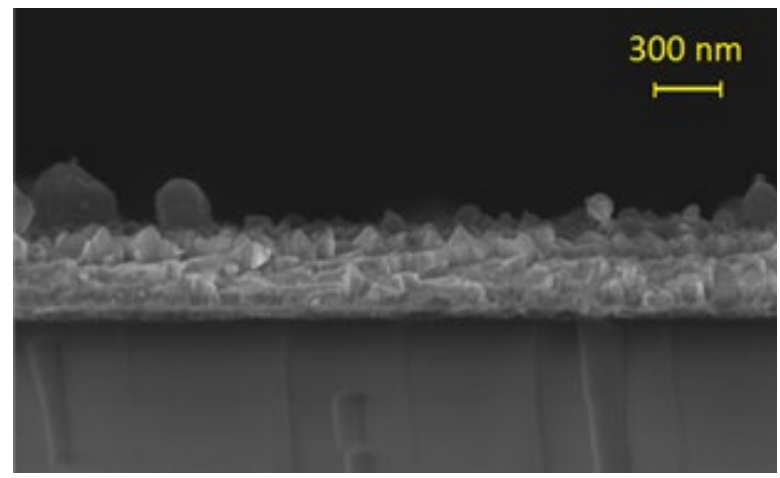

Figura 6. Imagen de la sección transversal del sustrato con la capa de bloqueo para 10 pasadas tomada por un MBE a una escala de $300 \mathrm{~nm}$. 


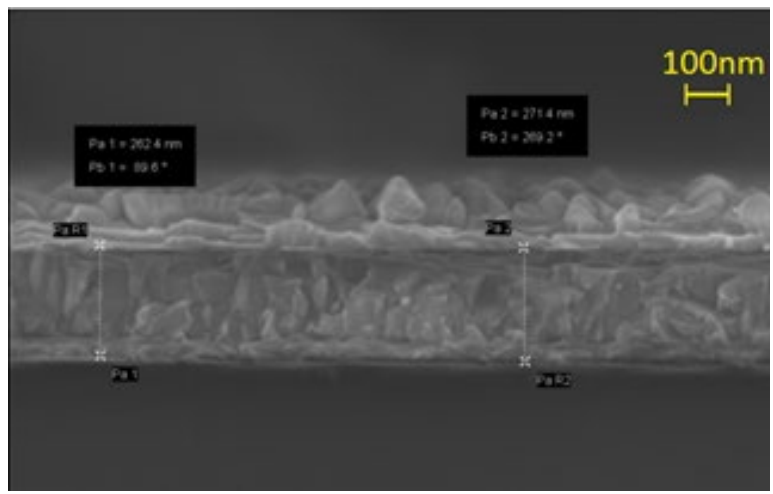

Figura 7. Imagen de la sección transversal del sustrato con la capa de bloqueo para 15 pasadas tomada por un MBE a una escala de 100nm.
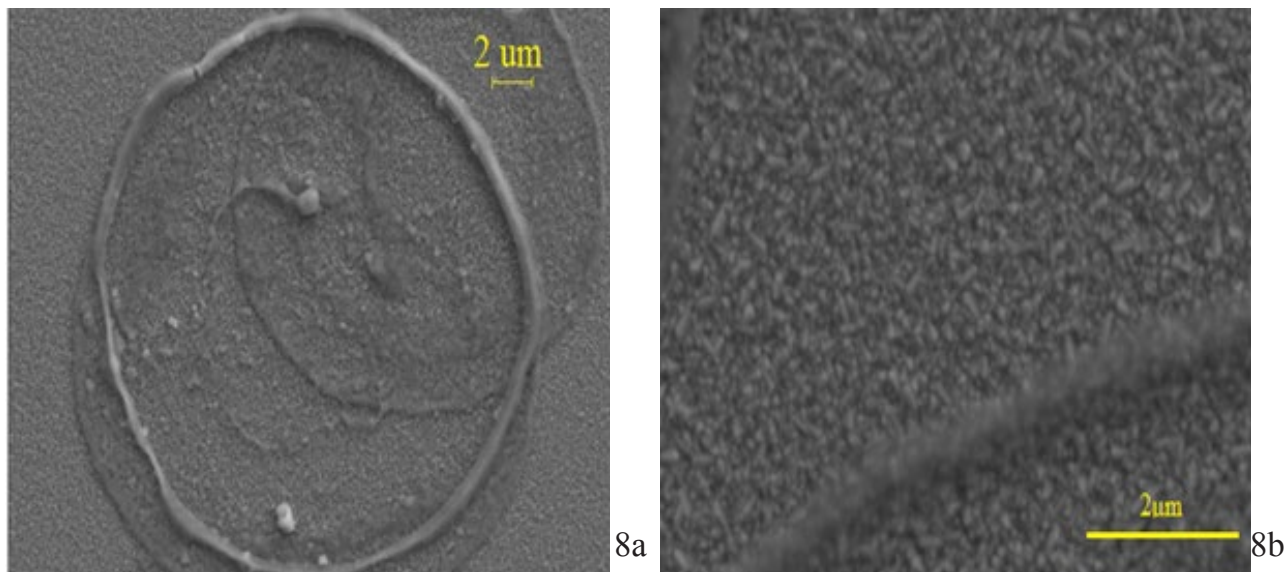

Figura 8. a) Fotografía de la superficie para de la capa de bloqueo de 10 pasadas, escala $2 \mu \mathrm{m}$. b) Fotografía de la superficie de la capa de bloqueo para 10 pasadas, escala de $2 \mu \mathrm{m}$. 


\section{Caracterización de la curva voltaje versus corriente}

Esta caracterización sirve para determinar su eficiencia y el factor de forma de la celda Grätzel. También se observa la tendencia de la corriente eléctrica que se da por el número de capas de bloqueo en la curva I-V (figura 9)

Figura 9. Curva del voltaje (V) versus Corriente $(\mathrm{mA})$ de la celda Grätzel con capas de bloqueo de $0,2,6$ y 9 pasadas.
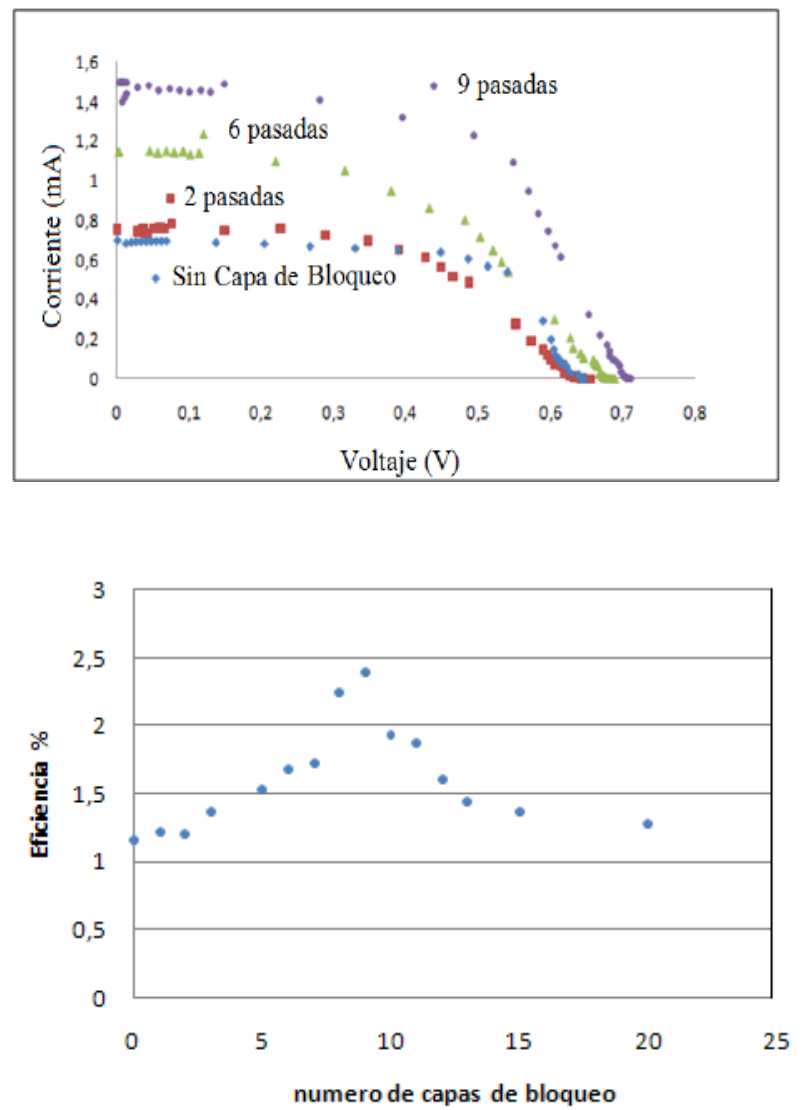

Figura 10. Resultado de la eficiencia obtenida versus el número de pasadas, donde se observa una mejor performance en la muestra para 9 pasadas de capa de bloqueo. 
Se ha hecho un estudio de una celda Grätzel con un colorante orgánico y capas de bloqueo con el fin de determinar el efecto de la capa de bloqueo dentro de una celda Grätzel con base en los resultados registrados en las me $\neg$ diciones de la celda solar construida; se pudo determinar algunas de las características. Por medio del MBE (ver figuras 6 y 7, sección transversal del sustrato y de la capa de bloqueo), se determinó que las capas de bloqueo para 10 pasadas tiene un espesor de 104,23 nm, mientras que las de 15 pasadas tienen un espesor de 153,2 nm, esto implica que por cada pasada se aumenta un espesor de $\sim 10 \mathrm{~nm}$ en promedio, con este resultado decimos que para 9 pasadas se tiene un espesor de $90 \mathrm{~nm}$, aproximadamente. Con respecto a la superficie (de las figura 8), se puede decir que una capa compacta, a partir del tamaño de granos, es de 34,5 nm de diámetro. Las figuras circulares que se forman en la superficie de la capa de bloqueo se deben al proceso del Rociado Pirolítico. En el figura9 se observa un ligero aumento en el voltaje de circuito abierto $\mathrm{V}_{\text {oC }}$; esto se debe a que el voltaje de circuito abierto depende del colorante, el electrodo y electrolito; como estos elementos fueron los mismo en todo las celdas la variación es insignificante, además de un mayor aumento en la corriente de corto circuito $\mathrm{I}_{\mathrm{SC}}$ en relación con el número de capas de bloqueo; este es un efecto directo de la capa de bloqueo evitando la recombinación del par electrón-hueco aumentando la corriente eléctrica en la celda; pero como se observa en la figura 10 este fenómeno sólo ocurre hasta 9 pasadas ó $\sim 90 \mathrm{~nm}$. Luego de esto, al aumentar el número de pasadas, hay una ligera disminución del $\mathrm{V}_{\mathrm{OC}} \mathrm{y}$ una disminución más pronunciada del $\mathrm{I}_{\mathrm{SC}}$, ocurriendo lo mismo con la eficiencia. De la figura 10 podemos concluir que la mejor eficiencia se obtiene para un número de pasadas igual a 9.

Se tiene referencia que el aumento del espesor de la matriz compacta se ha traducido en un aumento significativo en el factor de forma FF y la corriente eléctrica $\mathrm{I}_{\mathrm{SC}}{ }^{23}$. Tener en cuenta las nanodimensiones, es decir, el espesor de la matriz utilizada para el transporte de electrones desempeña un papel fundamental, ya que al aumentar el espesor hay un mejor bloqueo, pero al aumentar la longitud también aumenta el espacio donde se desplaza los electrones, aumentando la probabilidad que el electrón se recombine en su viaje al llegar al FTO. Es de señalar que el coeficiente de difusión de electrones es una función de la longitud de difusión y el tiempo de residencia de acuerdo con la correlación de la siguiente manera ${ }^{24}$ :

$$
L=\sqrt{D_{\theta} \tau_{\theta}}
$$

Donde $D_{e}=\frac{k T \mu_{e}}{e} \quad$ es decir $L \propto \tau_{e} \quad$ y $\quad L \propto \mu_{e}$

$L$ es la longitud de difusión, $D_{e}$ es el coeficiente de difusión de electrones, $\tau_{e}$ es el tiempo de residencia de electrones y $\mu_{\mathrm{e}}$ es la movilidad de electrones ${ }^{24}$. Un aumento de la longitud incrementa el transporte de electrones en la matriz de nanopartículas por el incremento de la movilidad electrónica, al mismo tiempo, aumenta el tiempo de residencia de electrones en la matriz. El aumento del espesor más allá del punto óptimo conducirá a mayor tiempo de residencia de electrones en la matriz de semiconductor que ponga en peligro el rendimiento DSC al facilitar la recombinación de electrones, ya sea con el electrolito o con el colorante y también el estado de energía del semiconductor.

Además, el tiempo de vida de electrones (tiempo de residencia) en el transporte de la matriz 
podría desempeñar un importante papel. Para igual tamaño de las nanopartículas de TiO2, la vida del electrón se ha encontrado que en $\mathrm{TiO}_{2}$ es de $0,6 \mathrm{~s}^{12}$. La residencia de electrones $\left(\tau_{\mathrm{e}}\right)$ en semiconductores se relaciona con $\mathrm{V}_{\mathrm{OC}}, \tau_{\mathrm{e}}$ aumenta con la disminución de $\mathrm{V}_{\mathrm{OC}}$, por la relación ${ }^{24,25}$ :

$$
\tau_{\theta}=-\frac{k T}{\theta}\left(\frac{d V_{o c}}{d t}\right)^{-1}
$$

Donde $k$ es la constante de Boltzmann, $T$ es la temperatura absoluta, y e es la carga elemental. Por lo tanto aumentando el espesor de película de nanomateriales podría facilitar para una la recombinación con el electrolito cuando se excede el espesor óptimo. El espesor óptimo tiene que ser diseñado sobre la base del rango espectral del colorante y del tipo de óxido metálico empleado en DSC.

\section{CONCLUSIONES}

Se ha presentado un estudio acerca de las celdas solares DSC y el uso de las capas de bloqueo obteniéndose lo siguiente:

Siendo la capa del semiconductor mesoporosa ( $\sim 50 \%)$ existen regiones donde no hay buen contacto entre el electrodo y el FTO. Con la capa de bloqueo (una capa más compacta) se mejora el contacto ayudando al transporte del electrón, además de evitar el contacto entre el FTO y el electrolito evitando, la recombinación del tipo:

$$
e_{\text {FTO }}^{-}+R^{+} \rightarrow R
$$

Se evaluó el rendimiento de la capa de bloqueo en la celda solar obtenido un eficiencia máxima para capa de bloqueo de espesor 90nm, aproximadamente.

En el laboratorio de Películas Delgadas de la UNI también se trabajó la celda Grätzel de óxido de titanio, pero sensibilizadas con un colorantes metal-orgánicos (N719), obteniendo una eficiencia de $2,1 \%{ }^{26}$, nosotros con las mismas condiciones usamos un colorante orgánico D7, más económico, pero gracias a las capas de bloqueo hemos obtenido una eficiencia de $2,39 \%$, superando a un colorante con mejor desempeño.

\section{AGRADECIMIENTO}

Un agradecimiento muy especial a la MSc. Julieta Cabrera Sotelo por la enseñanza brindada para preparar la solución de $\mathrm{TiO}_{2}$ por el método Sol Gel que se usó para la fabricación de capa de bloqueo, al Dr. Walter Estrada por las facilidades de trabajo en el laboratorio; a Carlos Ramos por la enseñanza sobre las celdas Grätzel, al Profesor Clemente Luyo por la disposición de tomar las fotos del MBE para analizar las muestras, al Ing. Oswaldo Rojas por facilitar los materiales y equipos en la toma de datos. Un agradecimiento especial al laboratorio Commossariat à l'énergie atomique et aux énergie alternatives. (Francia) por proporcionar las fotografías tomas por MBE que ayudaron a determinar el espesor de las capas de bloqueo y también la morfología de los mismos. 


\section{BIBLIOGRAFÍA}

1 Buchner, T.; Ewingen, N.H., Photosynthesis: Theory And Applications In Energy, Biotechnology and Nano $\neg$ technology. USA: Nova Science Publishers, 2009;56:1-41.

2 Gernot, R., Primary Processes of Photosynthesis: Principles and Apparatus. Rsc Publishing, 2008; 9: 61-63.

3 Jorge Ruelas M., Innovaciones Tecnológicas Utilizando Energías Renovables, Cusco, Perú 2010.

4 M.A. Green, J. Zhao, A. Wang, Progress and Outlook for High-Efficiency Crystalline Silicon Solar Cells, S.R. Wenham, Solar Energy Materials \& Solar Cells, 2001;65: 9-16.

5 Jianhua Zhao, Recent Advances of High-Efficiency Single Crystalline Silicon Solar Cells in Processing Technologies and Substrate Materials, Solar Energy Materials \& Solar Cells, 2004;82: 53-64.

6 Wu X, Keane JC, Dhere RG, DeHart C, Duda A, Gessert TA, Asher S, Levi DH, Sheldon P. 16.5\%- efficient CdS/CdTe polycrystalline thin-film solar cell. Proceedings of the 17th European Photovoltaic Solar Energy Conference, Munich, 2001; 2: 995 - 1000.

7 J. Pantoja Enríquez, X. Mathew, G.P. Hernández, U. Pal, C. Magan, D.R. Acosta, R. Guardian, J.A. Toledo, G. Contreras Puente, J.A. Chávez Carvayar, CdTe/CdS Solar Cells on Flexible Molybdenum Substrates, Solar Energy Materials \& Solar Cells,2004;82:307-314.

8 Brian O'Regan \& Michael Grätzel; "A low-cost, high-efficiency solar cell based on dyesensitized colloidal TiO2 films", Nature, 1991; 353: 737 - 740.

9 Tetsuo Soga, "Nanostructured Materials for Solar Energy Conversion", Institute of Technology Nagoya, Japan. 2006.

10 Tannia Marinado, "Photo electro chemical Studies Of Dye-Sensitized Solar Cells Using Organic Dyes” Royal Institute of Technology, Stockholm, Sweden. 2009.

11 Halme, J.; "Dye sensitized nanoestructured and organic photovoltaic solar cells technical review and preliminary test", University of Technology, Finland. 2002.

12 Thavasi V.; Renugopalakrishnan V.; Jose R.; Ramakrishna S. Controlled electron injection and transport at materials interfaces in dye sensitized solar cells. Materials Science and Engineering, 2008;R 63: 81- 99.

13 Stanley A., Verity B. and Matthews D., Solar Energy Materials \& Solar Cells, 1998; 52:141-154,

14 Daniel Hagberg, "Synthesis of Organic Chromophores for Dye Sensitized Solar Cells" Organic Chemistry, Royal Institute of Technology, Stockholm, Sweden. 2009.

15 Dennis Friedrich, "A study of charge transfer kinetics in dye-sensitized surface conductivity solar cells", Institut für Solare Brennstoffe und Energies peichermaterialien, Universität Berlin, German, 2011.

16 Changyun Jiang, Wei Lin Koh, Wei Hong, Yuning Li, "Influences of alcoholic solvents on spray pyrolysis deposition of $\mathrm{TiO} 2$ blocking layer films for solid-state dye-sensitized solar cells Journal of solid state chemistry,2013; 198:197-202

17 Quintana Cáceda, María Esther, Tesis de maestría, "Influencia del catalizador en la obtención de las fases cristalinas del óxido de titanio mediante el método del sol gel", Universidad Nacional de Ingeniería, Lima- Perú, 2004

18 Youl-Moon Sung, "Deposition of TiO2 Blocking layers of Photovoltaic cell Using RF 
Magnetron Sputtering technology", Energy Procedia, 2013;34:582-588.

19 Claudia N. Hoth, Pavel Schilinsky, Stelios A. Choulis, Srinivasan Balasubramanian and Christoph J. Brabec, Solution-Processed Organic Photovoltaics, Applications of Organic and Printed Electronics, 2013;180: 26-56.

20 Tatiana Nadezdina León Rojas, Tesis de Titulación: “Celdas Solares de Óxido de Zinc sensibilizadas con colorante”, Universidad Nacional de Ingeniería, Lima - Perú, 2012

21 M. Castro, J: Carpio, R. Guirado, A. Colmenar, L. Dávila, Energía solar fotovoltaica segunda edición, Editorial. PROGENSA, España, 2004.

22 María Esther Quintana Cáceda, Tesis Doctoral, "Celdas Solares de Óxido de Zinc Sensibilizadas con Colorante: Nuevos Enfoques”, Universidad Nacional de Ingeniería, Facultad de Ciencias, Lima - Perú, 2008.

23 Hyun-Joong Kim, Jae-Deok Jeon, D. Young Kim, ”Improved performance of dyesensitized solar cells with compact $\mathrm{TiO} 2$ blocking layer prepared using low-temperature reactive ICP-assisted DC magnetron sputtering", Journal of Industrial and Engineering Chemistry, 2012;18:1807-1812.

24 Juan Bisquert, * Francisco Fabregat-Santiago, Iva'n Mora-Sero', Germa` GarciaBelmonte, and Sixto Gimenez. Electron Lifetime in Dye-Sensitized Solar Cells: Theory and Interpretation of Measurements, J. Phys. Chem, 2009; 113: 17278-17290.

25 A. Zaban, M. Greenshtein, J. Bisquert, Determination of the Electron Lifetime in Nanocrystalline Dye Solar Cells by Open-Circuit Voltage Decay Measurements, Chem. Phys. Chem,2003; 4: 859-864.

26 Alexander R. Naupa Roque, Tesis de titulación, "Fabricación y caracterización de celdas solares sensibilizadas a base de dióxido de titanio nanoestructurado", Universidad Nacional de Ingeniería, Facultad de Ciencias, Lima - Perú, 2012 